\title{
A Necklace of Submandibular Stones: Case Report and Review of the Management of Submandibular Sialolithiasis
}

\author{
Nazish A. Khan ${ }^{1}$ \\ Richard James ${ }^{2}$
}

BDS, MFDSRCS, FFDRCSI

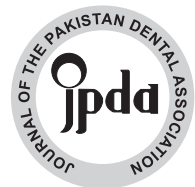

MBchB, BDS, LDSRCS, FRCS, FDSRCPS, FDSRCS, LLB

\begin{abstract}
AIM: To present an unusual case of 6 bilateral submandibular sialoliths and a review of the management of submandibular sialothiasis.

METHODOLOGY: Presentation of a case report of a 27-year-old gentleman with a 10-year history of bilateral recurrent submandibular sialolithiasis. A literature review of to assess the occurrence and management of submandibular sialolithiasis. RESULTS: The observance of a rare phenomenon of bilateral submandibular sialolithisasis, which only occurs in 3-5 $\%$ of cases.

CONCLUSION: The vast majority submandibular sialolithiasis occurs as single entities, multiple stones occur in only $3-5 \%$ of cases. Newer techniques have emerged for the management of this condition; however traditional methods are still being employed successfully.

KEY WORDS: Submandibular Silaolithiasis, Diagnostic Aids, Treatment Modalities.

HOW TO CITE: Khan NA, James R. A Necklace of Submandibular Stones: Case Report and Review of the Management of Submandibular Sialolithiasis. J Pak Dent Assoc 2017;26(4):196-98.

DOI: https://doi.org/10.25301/JPDA.264.196

Received: 29 October, 2017, Accepted: 29 December, 2017
\end{abstract}

\section{INTRODUCTION}

$\mathrm{S}$ ialolithiasis is a common condition affecting adult salivary glands, accounting for more than $50 \%$ of all salivary conditions. ${ }^{1}$

One of the post-mortem studies revealed calculi in the salivary glands in $1.2 \%$ of the population. ${ }^{2}$ The male to female predilection is $2: 1 .^{3}$

More than $80 \%$ occur in the submandibular gland or its duct, $6 \%$ in the parotid gland and $2 \%$ in the sublingual gland or minor salivary glands. ${ }^{4}$

Of the submandibular stones, $80 \%$ are intraductal and $20 \%$ are intraglandular. ${ }^{5}$

Submandibular sialoliths arise mainly due to the tortuous course of the Wharton's duct around the mylohyoid muscle and the increased concentration of calcium and mucin within the saliva, causing stasis. ${ }^{5}$

The vast majority of stones present as a single entity, with multiple or simultaneous stones, involving bilateral submandibular glands, being rare; $3-5 \%{ }^{6}$

We present a case report of six submandibular sialoliths involving the submandibular glands bilaterally.

\section{CASE REPORT}

A 27-year old gentleman was referred by his general

1. Specialist in Oral and Maxillofacial Surgery,James Cook University Hospital, UK. 2. Consultant Oral and Maxillofacial Surgeon, Norfolk and Norwich University. Corresponding author: "Dr. Nazish A. Khan" <aansa19@yahoo.com> medical practitioner to the Department of Oral and Maxillofacial Surgery at Norfolk and Norwich University Hospital. He presented with a 10-year history of dry mouth, difficulty swallowing and recurrent spontaneous expulsion of numerous stones from the floor of the mouth bilaterally. On examination, numerous stones were visible bilaterally through the oral mucosa on the floor of the mouth. A lower occlusal radiograph confirmed the presence of 6 concurrent bilateral submandibular stones, which appeared intra-ductal; therefore no further investigations were offered. (Figure 1).

The patient was consented for the removal of these stones under general anaesthetic. Five intra-ductal stones were removed (Figures 2 and 3 ).

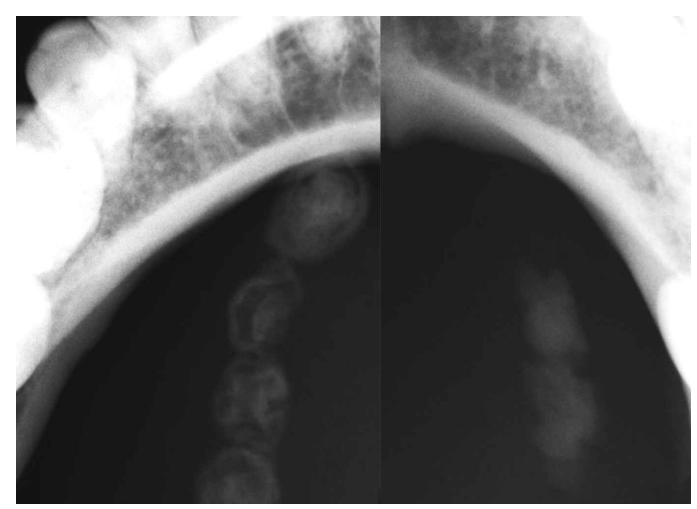

Figure 1: Pre-operative lower standard occlusal radiographs showing four stones within the right submandibular gland duct, and two with the left. 


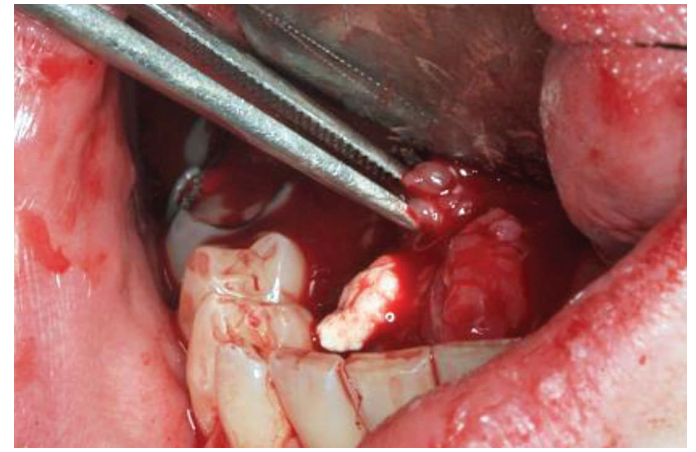

Figure 2: Surgical excision of the stones was performed via an intra-oral approach under a general anaesthetic; the tongue was held upwards to expose the stones, and an incision was made overlying them. Through blunt dissection, the stones were identified and retrieved.

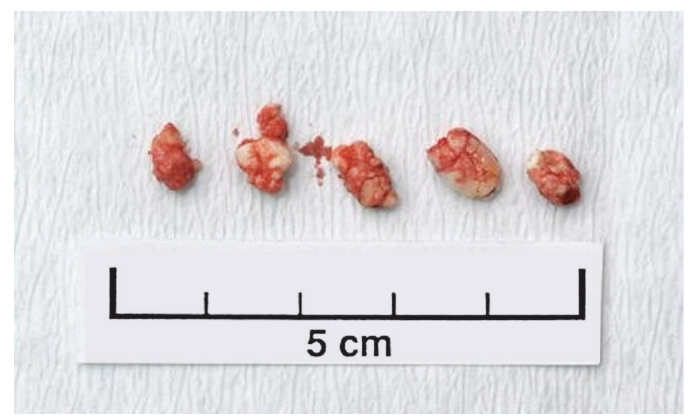

Figure 3: In total 5 stones were removed.

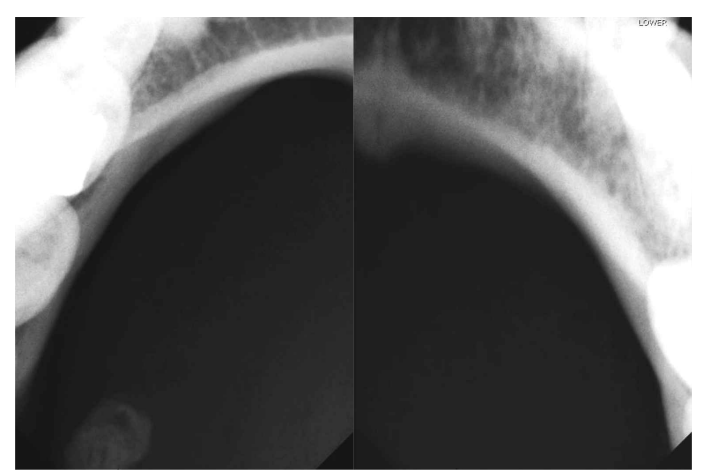

Figure 4: Post-operative lower standard occlusal views showing the remaining stone on the right at the hilum of the gland.

The 6th stone was embedded within the hilum of the gland, in close proximity to the lingual nerve, and was therefore left in situ (Figure 4). Upon reviewing the patient in clinic post operatively, there was no further stigma of dry mouth and the patient did not wish to be followed up in the future as his complaint had resolved.

\section{DISCUSSION}

Submandibular gland stones are a common presentation, constituting up to $85 \%$ of all salivary stones. ${ }^{7}$
They normally arise due to stasis within the tortuous Wharton's duct caused by the accumulation of organic cellular remnants and glycoproteins together with salts and other inorganic matter to form a nidus, or nest, which eventually calcifies. $^{3}$

The cardinal symptoms include pain and mealtime related swelling.

The stones take an elongated shape if they form within the duct, however if they form within the hilum of the gland, they can grow quite large and remain asymptomatic. ${ }^{7}$ Diagnosis is based on history and a thorough clinical examination. However sometimes submandibular stones can remain asymptomatic if the obstruction is not complete and saliva escapes around the calculi. ${ }^{8}$

Complete obstruction causes constant pain and swelling, pus may be seen draining from the duct and signs of systemic infection may be present. ${ }^{9}$

Special investigations can be undertaken to confirm the diagnosis and plan appropriate treatment.

Plain radiographs, especially intra oral lower occlusals are quite useful in locating radio-opaque stones. However, not all stones are radiopaque. Plain radiography visualises only $80-90 \%$ of submandibular stones and $60 \%$ of parotid duct stones, presumably due to differences in the composition of the secretion of the parent glands. ${ }^{10,11}$

CT scans are the more expensive, yet, the most accurate non-invasive technique described for localising stones. ${ }^{12,13}$

Sialography allows the whole duct system to be visualized, demonstrating calculi of all sizes and also glandular damage from chronic obstruction. ${ }^{4}$

Ultrasonography is well established in cases of clinical suspicion of sialolithiasis, it enables not only to visualise the stone, but also the gland. ${ }^{10,11}$

Treatment is dependent on the location and size of the stones. $88 \%$ of salivary calculi are reported to be less than $10 \mathrm{~mm}$ in size. ${ }^{14}$

In most cases of small stones, a trial of conservative treatment consisting of gland massage, hydration and salivary substitutes is adopted. ${ }^{15}$

If conservative measures fail and the stone is sufficiently far forward within the Wharton's duct, it can be milked forward, or alternatively sialolithotomy can be performed, by placing a trans oral incision directly on the stone. The stone is then grasped and removed and the gland milked. ${ }^{4}$

Stones located in the middle part of the Wharton's duct, posterior to the first molar, which cannot be palpated intra orally may require sialadenectomy. ${ }^{16}$

Newer techniques such as extracorporeal short-wave lithotripsy and endoscopic intracorporeal shockwave lithotripsy are also gaining popularity due to less damage to adjacent structures. ${ }^{17}$ 
The advent of salivary gland endoscopy has been a major advance, in providing an accurate means of diagnosing and locating intraductal obstruction, and also permitting minimally invasive surgery, which successfully relieves blockages not amenable to intraoral approaches. ${ }^{16}$

Submandibular gland excision is recommended in cases of substantial intra-glandular calculi, which are inaccessible via a trans-oral approach ${ }^{4}$ and also in cases where the gland has become fibrosed due to recurrent infections.

\section{CONCLUSION}

Submandibular sialolithiasis is a common presentation; however bilateral submandibular sialolithiasis is quite rare. Newer and more advanced techniques are on the horizon for accurate diagnosis and treatment of this condition. However, in this rare case of bilateral submandibular sialolithiasis, conventional methods were employed and the patient was treated successfully without any complications and resolution of his symptoms.

\section{CONFLICT OF INTEREST}

None declared

\section{REFERENCES}

1. Fowell C, MacBean A. Giant salivary calculi of the submandibular gland. J Surg Case Rep. 2012;2012:6. 2. Drage NA, Brown JE, Escudler MP, et al. Interventional radiology in the removal of salivary calculi. Radiol. 2000;214:139-42.

3. Cawson, Odell, Churchill Livingstone Essentials of Oral Pathology and Oral Medicine, 1998 6th edition Pages 239-240.

4. SJ Siddiqui, Sialolithiasis: An Unusually Large Submandibular Salivary Stone. Br Dent J. 2002; 193(2):
89-91.

5. Krishnappa, B.D Multiple submandibular duct [Wharton's duct] calculi of unusual shape and size. Indian J Otolaryngol Head Neck Surg. 2010 Jan;62:88-9.

6. Sunder V S, Chakravarthy C, Mikkilinine R, Mahoorkar S. Multiple bilateral submandibular gland sialolithiasis. Niger J Clin Pract 2014; 17:115-8.

7. Biddle, R.J. and Arora, S. Giant sialolith of the submandibular salivary gland. RadiolCase Rep 2008;1: $1-5$.

8. Satish Chandrashekhar, Ganeshan Submandibular Salivary Calculi, A case report, J dent Sci res 2001;16-18. 9. Pollack Jr CV, Severance Jr HW Sialolithiasis: case studies and review. J Emergency Med 1990; 8: 561-65. 10. Jäger L, Menauer F, Holzknecht N et-al. Sialolithiasis: MR sialography of the submandibular duct--an alternative to conventional sialography and US Radiol. 2000;216: 665-7.

11. Gritzmann N. Sonography of the salivary glands. Am J Roentgenol. 1989;153: 161-6.

12. Oteri G, Procopio RM, Cicciu M Giant Salivary Gland Calculi (GSGC): Report of two cases. Open Dent J 2011; 5:90-5.

13. Weissman JL Imaging of the salivary gland. Semin Ultrasound CT MR 1995; 16: 546-68.

14. Lustman J, Regev E, Melamed Y Sialothiasis: a survey on 245 patients and review of the literature. Int J Oral Maxillofac Surg 1990; 19: 135-8.

15. Williams MF Sialolithisis Otolaryngologic Clin North Am 1999; 32: 819-834.

16. Robert Witt, Sialolithiasis: Traditional and Sialoendoscopic Technique, The Open Access Atlas of Otolaryngology, Head \& Neck Operative Surgery, 2013. 17. Iro H, Schneider HTh, Fodra C et al. Shockwave lithotripsy of salivary duct stones. Lancet 1992; 339: 13331336. 